\title{
Assessing Support Requirements for Multidisciplinary Team Meetings
}

\author{
Bridget Kane Ken O’Byrne Saturnino Luz \\ Department of Computer Science \\ Trinity College Dublin \\ Dublin 2, Ireland \\ Department of Oncology, \\ St James's Hospital \\ Dublin 8, Ireland
}

$\{$ kanebt,obyrneke,luzs $\} @$ tcd.ie

\begin{abstract}
This paper profiles multidisciplinary team activity (MDT) in a typical teaching hospital setting and reports on a survey conducted among the teams to establish the information needs and constraints which affect their interaction. Support for pre-meeting work and post-meeting responsibilities is considered important in enabling the interaction at team meetings to be effective. Acoustics in the meeting room have higher priority over visual displays, although both 'hearing the discussion' and 'seeing images and colleagues' is important at meetings. Issues of time include scheduling and timing and are difficult to manage, particularly when individual roles belong to several MDTs and span more than one hospital. The potential is identified for automatic processing of some decisions by simple algorithm, which potentially will allow for more time at meetings to discuss complex patient cases in more detail.
\end{abstract}

\section{Introduction}

The aim of this paper is to identify how support for collaboration in multidisciplinary team meetings (MDTMs) can be improved in the context of developments in a large teaching hospital over recent years. We review current multidisciplinary team (MDT) practices at the hospital, provide a profile of the activities, and identify areas where technology can be usefully applied.

All of MDT members in the hospital were surveyed to identify common needs and constraints which may impact on the effectiveness of MDTs and MDTMs. The work follows earlier studies that analysed the work and use of technology at these meetings [2, 3, 4].

We expect that in the long term the results of this study will form the basis of user requirements specifications for systems aimed at supporting different aspects of MDT activity. This paper therefore focuses on the needs of individual participants, particularly in the context of their role, but it also takes account of resource implications for the hospital. Targeting such needs in the context of healthcare resources allows for a prioritization of requirements that will have greatest impact on hospital efficiency. Our survey allows for analysis based on time and facilitates comparison between different specialist roles and MDTs in the hospital. The impact of MDT meetings (MDTMs) is discussed from both the individual participant's role, and the hospital perspectives.

Over recent years there has been an expansion of MDT practice in hospitals because of the development of multidisciplinary teamworking and the number of clinical practice guidelines that recommend that patients, particularly cancer patients, are managed through a MDT. Over the past 20 years we have seen a shift in emphasis from a patient being managed by an individual doctor or team, to MDTbased service provision. Key performance indicators (KPIs) in modern hospitals typically include i) the number of cancer patients being managed by a MDT, and ii) if an individual patient has had the benefit of a MDT discussion. As part of the development of MDT service, there has been a marked increase in the demand for MDTMs. However, there are difficulties in managing this growth in demand for more specialised MDTs, and providing the resources necessary for sub-specialisation. Time [9] and Place [8] are identified constraints, and while technology has sought to facilitate synchronous and asynchronous collaboration and teamworking over multiple locations, there remain issues with respect to MDT working in modern hospitals that deserve to be addressed.

\section{Methodology}

The focus of this study is to identify the self-perceived issues among MDT members that are impacting the performance of the MDTMs. Each of the 10 MDTs in the hospital was surveyed. The cognate MDTs are based on either i) anatomic organ i.e. lung, breast, skin, ii) biologic system i.e. gastro-intestinal system, gynaecology and urology, iii) 
disease type i.e. lymphoma, haematology and iv) anatomic region i.e. head, neck \& thyroid. Almost 200 people were surveyed. There were 169 responses, (over $80 \%$ response rate).

These results are part of a larger on-going study on MDT working and MDTMs. Long-term ethnographic studies inform this research. Earlier work [2] focussed primarily on one MDT and our study is now expanded to include all of the MDTs in the hospital. Survey questions sought the views of team members with regard to issues affecting patient case discussions (PCDs) and MDTM performance. The questions were developed following over 100 hours of observation at MDTMs with up to 10 MDTs. Draft surveys were piloted with samples of individuals. Informal and semi-structured interviews with 16 MDT members, were conducted to check observations, interpretations and explore particular issues in more depth. All MDT members were encouraged to comment freely. The number of hours of preparation for meetings for each role, speciality, team and function was recorded. The individual's experience in working in MDTs was also noted.

\section{Results}

Table 1 is a breakdown of responses. Of the 169 respondents, approximately $25 \%$ belong to more than one MDT. There is considerable overlap in the Lymphoma and Haematology MDTs, and in Skin and Dermatology MDTs. The membership of these MDTs is combined in Table 1. See also section on 'Scheduling and Timing' and Table 3. Selfreported issues affecting members of the MDTs are listed in Table 2. The identified issues are categorised as involving: i) the selection of cases for the MDTM agenda and the distribution of the agenda list prior to the MDTM, ii) timing and scheduling of MDTMs, iii) supporting interaction during the MDTM, iv) the record of the MDTM discussion. In Table 3 the numbers of MDTs to which individuals are subscribed is given. Over $70 \%$ of respondents belong to one MDT, $16 \%$ are members of between 3 and, up to, 10 MDTs. Table 4 shows the time spent in preparation by individuals, depending on their role on the MDT. Almost $40 \%$ the preparation time for MDTMs is spent by the most senior role on the MDT.

The Agenda: Patients are identified manually for inclusion on the agenda for discussion by one of the MDT members, usually a senior clinical member, i.e. the doctor who has met the patient in a clinic, or conducted a procedure on the patient to be discussed. A number of particular issues are identified in Table 2, and discussed here, with respect to the selection of patients and the circulation of the agenda.

Timing: In order to have a comprehensive PCD specialists need to prepare for the MDTM. The greatest burden in preparation falls to radiologists and pathologists who re-
Table 1. Respondents per MDT and Speciality within the MDT.

\begin{tabular}{ll|ll}
\hline MDT & \multicolumn{3}{l}{ Speciality } \\
\hline Head, Neck & 28 & Physician & 39 \\
\& Thyroid & 34 & Surgeon & 46 \\
Lung & 20 & Pathologist & 10 \\
Gynaecology & 27 & Radiologist & 12 \\
Breast & 39 & Medical Oncologist & 11 \\
Gastro-Intestinal & & Radiation Oncologist & 12 \\
Lymphoma & 27 & Nurse & 22 \\
/Haematology & 11 & Speech \& Language & 3 \\
Skin /Dermatology & 12 & Therapist & 1 \\
Urology & 23 & Dietician & 1 \\
& & Physiotherapist & 2 \\
& & Medical scientist & 5 \\
& & Data Manager & 1 \\
\hline Total & 209 & Studen (admin) & 4 \\
\hline
\end{tabular}

view all material on a patient case prior to the MDTM. It is not unusual for materials such as radiological imaging studies or biopsy material to have been conducted at another hospital, and these items must be identified, located and reviewed prior to the MDTM discussion. Endoscopists will also have important information that needs to be compiled in advance of the PCD, and sometimes images taken during the procedure will need to be located in order to be made available to the MDTM. Organising these materials for review and to have the items available for the PCD requires staff to co-ordinate the information within each department. In radiology and pathology departments this resource can represent a significant proportion of staff time.

The agenda must be circulated in time for MDT members to be informed so that the necessary review of materials takes place prior to the MDTM. Reviewing material in pathology and radiology in advance of the MDTM saves time at the meeting and also allows for radiologists and pathologists to give a considered professional opinion (as recommended practice [1]). Both specialities dislike giving opinions on images presented at the MDTM without prior examination, and believe it to be a risky practice.

Volume of Cases per Agenda: The number of cases to include on an agenda is an unresolved question to date. The specialities who have the burden of preparation in advance of the meeting (radiology and pathology) sometimes express a desire to have a limit on the number of cases being included for a single MDTM. The clinical staff argue that it is not possible to set limits because clinical practice guide- 
Table 2. Self-reported issues affecting MDT performance

\begin{tabular}{ll}
\hline The Agenda & Timing \\
& No. of Cases per MDTM \\
& Reason for inclusion on agenda \\
& Case types \\
& Up-dating /checking agenda \\
\cline { 2 - 2 } The Meeting & Scheduling \\
& Timing \\
& Audio \\
& Visibility \\
& Videoconferencing \\
& Attendance \\
\cline { 2 - 2 } Record-keeping & Summary of discussion \\
& Availability in EPR \\
& Worklists \\
& Audit information \\
& Information for local practitioners \\
& National Data Repositories \\
\hline
\end{tabular}

lines that say all patients in a particular category should be discussed. Furthermore clinical staff report a benefit from MDTM discussion and reserve the right to include a patient on the agenda whenever they believe the patient will benefit.

Reason for discussion: The MDT member requesting a patient on an agenda knows the particular reason for discussion, but other MDT members report that the reason for discussion is often unclear. Depending on the patient's location in the care pathway, the particular question may differ. As examples: i) a new patient to the hospital who has had a preliminary diagnosis elsewhere may require an in-depth review of available material to be conducted to establish the definitive diagnosis and disease stage. ii) A patient who has undergone surgery will require to be reviewed for confirmation of pathological diagnosis (predicted prior to operation) and to query the possibility of residual disease. In the first example, radiological imaging studies and pathology from elsewhere will need to be located and reviewed prior to the MDTM. In the second example, radiological imaging postsurgery will require to be reviewed and compared to presurgical imaging; the pathologist will give special attention to surgical margins and compare diagnosis in the pre- and post-surgical material. The 'surgical margin' is the cut surface of the tissue removed from the patient. If a tumour is fully excised then this 'margin' is clear of tumour and the abnormal tissue examined by the pathologist is surrounded by non-cancerous tissue. Both the pathologist and radiologist pay particular attention to the interpretation of the material in the context of the patient having had surgery, or a clinical diagnosis in example i) above. In the first example, the pathology opinion on the tumour type is the most important question, as this will be the primary influence in
Table 3. The number of MDTs to which individual are subscribed

\begin{tabular}{ccc}
\hline $\begin{array}{c}\text { Number of MDTs } \\
\text { to which subscribed }\end{array}$ & $\begin{array}{c}\text { Number of } \\
\text { Individuals }\end{array}$ & $\begin{array}{c}\% \\
\text { Respondents }\end{array}$ \\
\hline 1 & 121 & 72 \\
2 & 20 & 12 \\
3 & 7 & 4 \\
4 & 2 & 1 \\
5 & 2 & 1 \\
$6-10$ & 17 & 10 \\
\hline Total & 169 & 100 \\
\hline
\end{tabular}

determining the treatment approach, i.e. chemotherapy or surgery. The question facing the MDT in the second example will be "is more surgery needed, or is chemotherapy or radiation therapy appropriate now?" Indeed, pathology is regarded as a quality check on a surgical procedure. Knowing the reason for the discussion and having relevant clinical information, is particularly important for pathologists and radiologists when reviewing images for discussion at the MDTM. Both radiology and pathology give interpretation in the context of the information provided. They can provide a more focussed opinion, (which may take less time than a full review), when they have all the relevant information and know the reason for the discussion at the MDTM.

Cancer cases vs. Non-cancer cases: Cancer practice guidelines tend to recommend that all cancer patients should be managed by a MDT. Some specify that patients suspected of cancer should be managed by the MDT. MDT discussion and management is also recommended in many complex diseases [11]. Furthermore, many clinicians consider the MDTM to be an important teaching and learning forum where the team will benefit from the presentation and review of 'interesting' non-cancer patient cases.

The question of 'who suspects?' has been raised with respect to breast cancer in particular and merits special consideration. The management of patients with breast cysts is emerging as a special case and will likely lead to change in practice guidelines in the future. Currently the recommendation is for all patients with suspected breast disease, (including cysts), to be assessed by three modalities namely clinical assessment, radiology and pathology and that these three investigative modalities should be correlated at a MDTM [7]. Where results are not concordant, further procedures must be undertaken. However, clinical assessment by experienced staff can identify most benign breast cysts, the ultrasound investigation can be straightforward and the aspiration material from a benign breast cyst has certain microscopic characteristics. It can be argued that when these three independent modalities are fully concordant that there is no need for discussion at a MDTM and 
Table 4. Amount of time spent by individuals per week in preparation for MDTMs, by Role

\begin{tabular}{|c|c|c|c|c|c|c|c|c|c|}
\hline \multirow[t]{2}{*}{ Role } & \multicolumn{7}{|c|}{ Hours of Preparation per week } & \multirow{2}{*}{$\begin{array}{c}\text { Total } \\
\text { People }\end{array}$} & \multirow{2}{*}{$\begin{array}{c}\text { MINIMUM } \\
\text { Role Hrs }\end{array}$} \\
\hline & Nil & $\approx 1 \mathrm{hr}$ & $1-2$ & $2-3$ & $3-4$ & $\gg 4 \dagger$ & Unknown & & \\
\hline Consultant & 3 & 21 & 14 & 4 & 1 & $9 \ddagger$ & 1 & 53 & 101 \\
\hline Snr Registrar & 3 & 6 & 2 & 3 & 1 & - & - & 15 & 23 \\
\hline Registrar & 4 & 14 & 16 & 5 & - & 1 & 2 & 42 & 65 \\
\hline $\mathrm{SHO}$ & - & 1 & 1 & - & 1 & 1 & - & 4 & 11 \\
\hline Intern & 1 & 6 & 3 & 1 & 1 & - & - & 12 & 19 \\
\hline Nursing & 7 & 10 & 3 & 5 & - & 2 & - & 27 & 39 \\
\hline Administration $*$ & $=2$ & 2 & - & - & 1 & - & - & 5 & 6 \\
\hline Allied Health & 4 & 1 & 2 & - & - & - & - & 7 & 3 \\
\hline Student & 3 & - & 1 & - & - & - & - & 4 & 2 \\
\hline \multicolumn{6}{|c|}{$*$ Does not include 4 full-time administrative staff } & \multicolumn{4}{|c|}{$\begin{array}{l}\text { TMany emphasise much more than } 4 \text { hours per week } \\
\text { †One consultant reports } \leq 8 \text { hrs per week for } 1 \text { MDTM }\end{array}$} \\
\hline Total people & 28 & 61 & 41 & 16 & 4 & 13 & $\begin{array}{ll}3 & 1\end{array}$ & 69 peopl & \\
\hline $\begin{array}{l}\text { Total hours } \\
\text { (People } \cdot \text { Hours) }\end{array}$ & $28 \cdot 0$ & $61 \cdot 1$ & $41 \cdot 2$ & $16 \cdot 3$ & $4 \cdot 4$ & $13 \cdot 4$ & $3 \cdot 0$ & & $\gg 269$ hours \\
\hline
\end{tabular}

that the cases could be managed through a simple algorithm. All three assessment modalities utilise a 5-point scale with a prefix $\mathrm{C}$, R or $\mathrm{P}$ representing Clinical assessment, Radiology and Pathology respectively. The scale 1, 2, 3, 4 and 5 is used to denote 'unsatisfactory', 'benign', 'suspicious', 'malignant' and 'invasive' classification. Thus, a patient with a benign breast cyst would have results of $\mathrm{C} 2, \mathrm{R} 2$ and $\mathrm{P} 2$ and no further follow-up is considered necessary. In a medium sized breast cancer centre the number of patients presenting with breast cysts, all benign for breast cancer, can be 20 50 per week. Currently these patients are reviewed quickly at a MDTM in compliance with current practice guidelines.

While the focus for many MDTM is on cancer patient management, non-cancer cases are also discussed at MDTMs, particularly in complex diseases such as inflammatory bowel disease [11], or T.B.. Although MDT input is considered useful in many such illnesses, some specialities such as chemotherapy have little contribution, if any, in non-cancer cases. (This is not universally true. Radiation or chemotherapy therapy can be used in the treatment of some non-cancer disorders.) For this reason some MDTs, e.g. breast, have organised their MDTM agenda and cases are prioritised into i) those that have full pathology and full radiology available and are post-surgery, ii) those patients that have undergone pathology sampling and radiology and on whom surgical decision is awaited, iii) those who have undergone radiology assessment alone, and iv) those who have had clinical assessment and believed to be (clinically) benign. This last category contains mostly cystic lesions and the radiation and medical oncologists are free to leave the MDTM, if they choose.

Updating Agenda: Two issues have emerged with respect to updating information for the agenda: i) requesting a PCD and ii) updating a request.
Currently an individual team member completes and online form to request a patient for inclusion on the MDTM agenda. This request is processed by a MDT co-ordinator who locates external radiology and/or pathology, etc. if necessary for review, and the agenda is circulated to the MDT once all the material is available. Each MDT has a cut-off time for inclusion of a patient on the agenda for the next MDTM, to allow for each of the team to make the necessary preparation for an adequate discussion. For example, for a Monday morning meeting at 0800, the agenda is circulated to the MDT by Thursday lunchtime. All requests for case discussion need to be submitted to the MDT coordinator by Thursday morning. MDT members report that it can be difficult for one member know if a colleague has already submitted the on-line form, or to know how many cases are already requested for the MDTM. Neither is it possible to add new information, once the on-line form is submitted and the agenda is circulated. Because of the nature of medical work, the patient situation is continuously changing and it is desirable for radiology and pathology in particular to have all necessary information available while reviewing material.

Submissions entered in duplicate for discussion are managed manually by MDT co-ordinators and a second or subsequent request for PCD is deleted. It has been proposed that inputted information for the agenda can be used as a basis for the final report, outcome or record, of the PCD. However that information may be out-of-date by the time the patient is discussed and the update is provided in talk at the meeting. Methods to update patient information are currently under consideration.

For all MDTs, it is acknowledged that urgent cases may arise following the circulation of the agenda, and it is not uncommon for several revised agendas to be circulated fol- 
lowing the 'cut-off' time, up to the day before the MDTM.

The Meeting: Supporting interaction during the MDTM where different specialists give their professional opinion on cases while referring to patient imaging and other data, is a challenge. The biggest issues reported relate to time, hearing colleagues, seeing images on display, and seeing other MDT members especially when videomediated.

Scheduling and Timing: The time issues reported relate to i) the problem of finding time for the team to meet within busy schedules, ii) having enough time for preparation beforehand and iii) having sufficient time for discussion at a meeting with all of the necessary specialists present.

Often the only time available where all can meet is in the early morning, or at lunchtime. Efforts are on-going to incorporate the MDTM into the normal working day, but scheduling operation theatres, endoscopy sessions and out-patient clinics so that all the MDT members are available for the MDTM is a challenge. Furthermore many MDT members have responsibilities to more than one hospital, and so several hospitals may have to synchronise their schedules and timetables for procedures or clinics. This is not always possible. While videoconferencing may suggest that some of these constraints of time and place may be facilitated, it has not proven to be a satisfactory solution to date. Even for the 121 individuals who belong to one MDT (Table 3 and 5) it can prove a challenge to organise schedules for the MDTM to take place. Typically the specialisms whose contribution spans several MDTs are radiology, pathology, medical and radiation oncology and some surgical roles (such as plastic surgery). Significant resources are required to support provision of specialists in multiple MDTs. Videoconferencing is found to be helpful for medical oncology services in particular, as the specialist is often located in a smaller regional centre.

Audio and Visual Displays: Interaction is conducted through talk, supplemented with gaze and gesturing, and occasional pointing, annotation or drawing of anatomical structures. Thus, both audio and visual interaction devices can be usefully applied.

Over $70 \%$ of MDT respondents report difficulty in hearing colleagues in the same room from time to time. Two rooms are used for most MDTMs, and there is no statistically significant difference between venues. Senior MDT members, all active vocal participants, usually announce their difficulty in hearing their junior colleagues. However, they are less likely to announce difficulty in hearing their peers. Junior staff very rarely acknowledge in public that they cannot hear a senior MDT member, and usually confer with a colleague, or check afterwards, on facts they require.

It has been shown useful to have information available during a PCD both as an aide memoire during the PCD and also to facilitate checking, or rechecking for individual team members as the PCD progresses. In the absence of a plasma
Table 5. Frequency for individuals in the number of MDTMs attended per week

\begin{tabular}{lcc}
\hline $\begin{array}{l}\text { Number of meetings } \\
\text { attended per week }\end{array}$ & $\begin{array}{c}\text { Number of } \\
\text { Individuals }\end{array}$ & $\begin{array}{c}\% \\
\text { Respondents }\end{array}$ \\
\hline$<1$ & 7 & 4 \\
1 & 86 & 51 \\
1.5 & 6 & 4 \\
2 & 27 & 16 \\
2.5 & 4 & 2 \\
3 & 19 & 11 \\
3.5 & 1 & 1 \\
4 & 14 & 8 \\
5 & 1 & 1 \\
6 & 2 & 1 \\
10 & 1 & 1 \\
Unknown & 1 & 1 \\
\hline Total & 169 & 101 \\
\hline Fractions represent meetings attended less than once weekly \\
i.e. MDTMs held fortnightly / monthly
\end{tabular}

screen display, information can be projected using a ceiling mounted projector. However, the noise of the projector's fan, can be sufficiently distracting that MDT members choose to turn off the projector in order to hear fellow MDT colleagues more easily. The sound of the air conditioner in one of the rooms has also been reported to detract from the acoustic quality in the room. Microphones are not currently in use in the meeting rooms. Investment in audio enhancement is under active consideration.

Videoconferencing: Some MDTs connect with remote hospitals during MDTMs to discuss patient cases, using a videoconferencing system. While videoconferencing saves the patient having to travel to the large specialist centre for assessment, and allows the MDT to extend over a wide geographical area, some difficulties have been experienced which have resulted in the service not being developed as rapidly as one might expect. The most critical issue is time and timing. Discussion in videoconference takes more time than a co-located discussion [2] and the time constraint has not encouraged the MDT to engage in developing more linkages with other centres.

Attendance: Facilitating all of the MDT to be present for the meeting is vital for a full PCD. It is also important that attendance at the MDTM is recorded for two reasons. Firstly, attendance of a speciality during a discussion, but without active involvement, is understood by MDTs to mean that the input of that speciality is unnecessary. Secondly, a record of MDTM attendance is considered necessary to help ensure that only those individuals who are authorised to attend are present. This requirement provides a safeguard for respecting patient privacy and maintaining confidentiality within the MDT. All staff are contractually 
bound to respect the patient's right to privacy and the hospital's responsibility towards the patient. Reinforcing this responsibility through a formal record of MDTM attendance is considered helpful and complies with hospital policy.

Record-Keeping: Having a record of the patient case discussion that will be available in the Electronic Patient Record (EPR) is a priority for MDTs. Such a record is also required to serve clinical audit and quality management functions as well as providing information for $\mathrm{Na}$ tional repositories such as the National Cancer Registry and Department of Health level statistics. The MDTM is identified as a useful forum for gathering information for a range of purposes that would otherwise need to be gathered through labor intensive processes. Across all MDTs surveyed, certain items of information are considered essential as a PCD record. The record of the final decision and follow-up tasks is considered fundamental. However, the rational for the decision, the contributors to the discussion and the options considered are also desired. Without an audio-visual record of the PCD, these items cannot be readily captured. One of the limiting factors in the development of an audio-visual MDTM record is the difficulty in retrieval of information afterwards and its integration into the EPR. While research on analysis of MDTM recordings is on-going $[10,5]$, further work is needed for all of the issues to be satisfactorily resolved.

In the short term, the real time recording of opinion and facts during meetings is being implemented. Some UKbased MDTs are known to have real time data entry on overhead plasma screens of key items of information [6]. By having the data entry visible to the MDT, any misunderstandings can be corrected and errors avoided. Reservations by the MDTs to implement such a system have been expressed. The main concern is that it will take more time and create inefficiencies. Other concerns involve the items to be included in a record, and potential difficulties in implementing new technology within already overstretched schedules. The discussion continues, but there is general agreement that to develop the model of MDTM further, a more satisfactory method of recording the decisions taken at the MDTM is necessary.

\section{Conclusion}

Supporting collaboration at MDTM setting can be considered at several levels, not only in the people-to-people interactions that take place during MDTM event. Examining issues of concern for team members in their work associated with their attendance at MDTMs will promote improved collaboration during discussion.

As MDT working increases in today's hospitals, and resources become more limited, there is a greater urgency for technological solutions to be identified that will enable the
MDT service to be delivered more effectively. Considering that a large amount of time is spent in preparation for MDTMs, as well as in the conduct of meetings, it is argued that support for preparation and engagement at meetings should be prioritised. The problem of scheduling is a real concern. The need for a formal record of proceedings is seen as the most important requirement however, as it directs day to day work for many specialists and provides a legal record of the decision that was agreed at the meeting.

\section{Acknowledgments}

A sincere 'thank you' is extended to all of the MDTs at St. James's hospital, Dublin who participated in this study. B. Kane is the recipient of an IRCSET Research Fellowship award under the IRCSET Enterprise Partnership Scheme with St. James's Hospital Board.

\section{References}

[1] Board of the Faculty of Clinical Radiology. Cancer multidisciplinary team meeting - standards for clinical radiologists. London, November 2005.

[2] B. Kane and S. Luz. Multidisciplinary medical team meetings: An analysis of collaborative working with special attention to timing and teleconferencing. Computer Supported Co-operative Work (CSCW), 15(5-6):501-535, Dec 2006.

[3] B. Kane and S. Luz. Achieving diagnosis by consensus. Computer Supported Cooperative Work (CSCW), 18(4):357 - 392, April 2009. DOI:10.1007/s10606-009-9094-y.

[4] B. Kane, S. Luz, D. S. O'Briain, and R. McDermott. Multidisciplinary team meetings and their impact on work-flow in radiology and pathology departments. BMC Medicine, 5(15), 2007.

[5] S. Luz. Locating case discussion segments in recorded medical team meetings. In SSCS '09: Proceedings of the ACM Multimedia Workshop on Searching Spontaneous Conversational Speech, pages 21-30, Beijing, China, Oct. 2009. ACM Press.

[6] E. J. Macaskill, S. Thrush, E. M. Walker, and J. M. Dixon. Surgeons' views on multi-disciplinary breast meetings. European Journal of Cancer, 42:905-908, 2006.

[7] N. O'Higgins. Developing quality care for breast services in Ireland, 2006.

[8] J. S. Olson, G. M. Olson, and D. Meader. Distance matters. In A. J. S. Kathleen E. Finn and S. B. Wilbur, editors, Video-Mediated Communication, chapter Face-to-Face Group Work compared to Remote Group Work with and without Video. ACM Press, 2000.

[9] M. C. Reddy, P. Dourish, and W. Pratt. Temporality in medical work: Time also matters. Computer supported Cooperative Work (CSCW), 15(1):29-53, 2006.

[10] J. Su, B. Kane, and S. Luz. Automatic meeting participant role detection by dialogue patterns. In Proceedings of COST 2102 Int. School - Development of Multimodal Interfaces: Active Listening and Synchrony, volume 5967, 2010.

[11] A. Windsor and A. Forbes. The inflammatory bowel disease unit and the multidisciplinary team meeting, July 2007. 